\title{
Spline Approximation to the Solution of the Volterra Integral Equation of the Second Kind
}

\author{
By Arun N. Netravali
}

\begin{abstract}
A cubic spline approximation in $C^{2}$ to the solution of a general Volterra integral equation of the second kind is constructed. Under certain conditions, convergence of the approximation and its first two derivatives is proved and error bounds are obtained. The question of stability is not examined.
\end{abstract}

1. Introduction. The developments in the present paper on the cubic spline approximation to the solution of the Volterra integral equation of the second kind

$$
x(s)-\int_{0}^{s} K(s, t, x(t)) d t=y(s), \quad 0 \leqq s \leqq b,
$$

complement those in a preceding paper [1] on the Fredholm integral equation. In the above equation, $K(\cdot, \cdot, \cdot)$ and $y(\cdot)$ are real-valued functions on subsets of $R^{3}$ and $R^{1}$, respectively, satisfying appropriate smoothness conditions. The function $x(\cdot)$ from the subset of $R^{1}$ into $R^{1}$ denotes the solution of (1).

Several authors have investigated the numerical solution of (1) using various methods which rely on the approximation of the integral appearing in (1) by some quadrature. Use of the trapezoidal rule for the case of convolutional kernels for linear equations was discussed by Jones [2], and for general kernels by Noble [3]. Lobatto quadrature schemes were used by Jain and Sharma [4]. Such methods were also discussed by Fox and Goodwin [5]. A systematic study of a class of such methods was undertaken by Linz [6] who generalized some previous approaches and gave a complete error analysis. Runge-Kutta methods were developed in a manner similar to that of ordinary differential equations by the use of Taylor's expansion, by Pouzet [7], [8], Laudet [9], Bel'tjukov [10], and Feldstein [11].

All the methods mentioned above give approximate values of the solution at a set of discrete points. Our aim is to obtain a technique which gives rise to globally convergent methods having several continuous derivatives. These methods are useful when we have a priori knowledge of the smoothness properties of the kernel $K(s, t, x)$ and the forcing function $y(s)$, since, if the kernel and the forcing function have continuous derivatives up to the order $p$ in the domain of integration, then the solution also has continuous derivatives up to the same order. They are economical when the value of the solution and its derivatives are required at a large number of points where usual methods of computation [to compute at each point] would be time consuming.

Such methods have been discussed recently by Loscalzo and Talbot [12] for the solution of ordinary differential equations and were later extended by Loscalzo and

Received June 14, 1971, revised January 18, 1972 and May 10, 1972.

AMS (MOS) subject classifications (1970). Primary 65R05; Secondary 65D99. 
Schoenberg [13], and Varga [14]. Hung [15] has recently discussed the use of spline functions for solving Volterra integral equations of first and second kind. In particular, for a Volterra integral equation of second kind, he obtains convergent solutions in $C$ and $C^{1}$ using linear and quadratic splines. He also shows that his technique, using cubic spline of full continuity [i.e. solutions in $C^{2}$ ], is divergent and therefore develops a method using Hermite interpolation to obtain a cubic spline solution in $C^{1}$, gives asymptotic error estimates, and proves $A$-stability in the sense of Dahlquist. Thus, his existing techniques cannot be used to obtain a solution in $C^{2}$ and if they are extended by using Hermite interpolation of higher degree, they will involve additional computation [i.e. computation of second or higher derivatives] as compared to our method. In this paper, we show that the divergence of higher degree splines depends upon the particular method used and is not necessarily a result of strictness of the continuity requirements. We develop a self-starting technique using cubic splines in $C^{2}$, prove its convergence, and give high quality error bounds for the solution as well as its first two derivatives. The problem of stability along with some other extensions will be discussed in a future paper.

2. Construction of the Approximation and the Error Bounds. Although we could consider an arbitrary mesh, for simplicity, we divide the interval $[0, s]$ into $m$ equal parts such that $m h=s$, and define the mesh by

$$
\Delta_{m}: 0, h, 2 h, \cdots, m h=s .
$$

We denote the cubic spline of type I [16, p. 75] which interpolates the values $x^{(1)}(0)$, $x(0), x(h), \cdots, x(m h), x^{(1)}(m h)$ of the function $x(t)$ by $S_{\Delta_{m}}(x, t), 0 \leqq t \leqq s$. Such a spline may be expressed in terms of the basis of cardinal splines $C_{m, j}(t), j=0, \cdots$, $m+2$, which are defined as type I cubic splines on $[0, s]$ satisfying

$$
\begin{aligned}
C_{m, j}(i h) & =\delta_{i i} ; \quad i=0,1, \cdots, m ; \quad j=0,1, \cdots, m, \\
C_{m, i}^{(1)}(0) & =C_{m, j}^{(1)}(s)=0 ; \quad j=1, \cdots, m, \\
C_{m, k}(i h) & =0 ; \quad k=m+1, m+2 ; \quad i=0,1, \cdots, m, \\
C_{m, m+1}^{(1)}(0) & =1 ; \quad C_{m, m+1}^{(1)}(m h)=0, \\
C_{m, m+2}^{(1)}(0) & =0 ; \quad C_{m, m+2}^{(1)}(m h)=1,
\end{aligned}
$$

where $\delta_{i j}$ is the Kronecker delta. Thus the type I cubic spline interpolating the solution $x$ of (1) on $\Delta_{m}$ may be written as

$$
S_{\Delta_{m}}(x, t)=\sum_{k=0}^{m} x(k h) C_{m, k}(t)+x^{(1)}(0) C_{m, m+1}(t)+x^{(1)}(m h) C_{m, m+2}(t)
$$

The values of the approximate solution, $\hat{x}$, obtained by replacing the integral in (1) by spline quadrature can be written as

$$
\begin{aligned}
\hat{x}_{m}(m h)=y(m h)+\int_{0}^{m h} K[m h, & t, \sum_{i=0}^{m} C_{m, j}(t) \hat{x}_{i}(j h) \\
& \left.+C_{m, m+1}(t) \hat{x}^{(1)}(0)+C_{m, m+2}(t) \hat{x}_{m}^{(1)}(m h)\right] d t,
\end{aligned}
$$




$$
\begin{aligned}
\hat{x}_{m}^{(1)}(m h)= & y^{(1)}(m h)+K\left(m h, m h, \hat{x}_{m}(m h)\right) \\
+ & \int_{0}^{m h} K^{(1)}\left[m h, t, \sum_{j=0}^{m} C_{m, j}(t) \hat{x}_{i}(j h)\right. \\
& \left.+C_{m, m+1}(t) \hat{x}^{(1)}(0)+C_{m, m+2}(t) \hat{x}_{m}^{(1)}(m h)\right] d t
\end{aligned}
$$

where

$$
\begin{aligned}
\hat{x}(0) & =x(0)=y(0), \\
\hat{x}^{(1)}(0) & =y^{(1)}(0)+K(0,0, y(0))
\end{aligned}
$$

and

$$
K^{(i)}(s, t, x)=\frac{\partial^{i}}{\partial s^{i}}[K(s, t, x)] .
$$

Equations (5) and (6) are two simultaneous nonlinear algebraic equations for $\hat{x}_{m}(m h)$ and $\hat{x}_{m}^{(1)}(m h)$, which can be solved by any of the available, sufficiently accurate, "root finding" techniques. Solvability of these equations and the error bounds are treated in the Theorem. The global approximate solution is obtained by simple cubic spline interpolation on $x(0), x^{(1)}(0), \hat{x}_{1}(h), \cdots, \hat{x}_{m}(m h), \hat{x}_{m}^{(1)}(m h)$, which we denote by $S_{\Delta_{m}}\left(\hat{x}_{m}, t\right)$.

We first state two lemmas whose proofs can be found elsewhere. From now on, $s$ is assumed to be a constant and $h$ is chosen such that $s=m h$.

LeMma 1. If $\left\{C_{m, j}(t) ; j=0,1, \cdots, m+2\right\}$ is the set of cardinal splines on the mesh $\Delta_{m}$, then there exist three positive constants $M_{1}, M_{2}, M_{3}$ such that

$$
\text { (a) } \int_{0}^{m h}\left|C_{m, j}(t)\right| d t \leqq M_{1} h ; \quad j=0, \cdots, m+2,
$$

and hence

$$
\begin{array}{ll}
\text { (b) } \max _{0 \leq t \leq m h}\left[\left|C_{m, j}(t)\right|\right] \leqq M_{2} ; & j=0, \cdots, m+2, \\
\text { (c) } \int_{0}^{m h}\left|C_{m, j}(t)\right|^{2} d t \leqq M_{3} \cdot h ; & j=0, \cdots, m+2 .
\end{array}
$$

Proof. For the cardinal splines $C_{m . j}(t), j=1, \cdots, m-1$, the above follows easily from Theorem 1 of [17]. For the remaining splines, it can be shown similarly that the conclusion still holds.

Lemma 2 (Gronwall; See Lees [18, Lemma 1]). Let $\left\{e_{n}\right\}$ be a sequence of real numbers satisfying

$$
\left|e_{n}\right| \leqq A \cdot h \cdot \sum_{i=0}^{n-1}\left|e_{i}\right|+B_{n}, \quad n=1, \cdots,
$$

for some positive constant $A$ and nondecreasing sequence $\left\{B_{n}\right\}$. Then

$$
\left|e_{n}\right| \leqq B_{n} \cdot \exp (A h n) .
$$

THEOREM. Let the solution of $(1), x(\cdot)$, be in $C^{4}[0, m h]$ and

$$
\left|K^{(i)}\left(s, t, x_{1}\right)-K^{(i)}\left(s, t, x_{2}\right)\right| \leqq L_{i}\left|x_{1}-x_{2}\right| ; \quad i=0,1 ; 0 \leqq t \leqq s \leqq b .
$$


Then

(16a)

$$
\max _{1 \leqq i \leqq m}\left[\left|x^{(i)}(j h)-\hat{x}_{j}^{(i)}(j h)\right|\right] \leqq O\left(h^{4}\right), \quad i=0,1,
$$

and there exist constants $M_{4}, M_{5}$, and $M_{6}$ such that

$$
\sup _{0 \leqq t \leqq m h}\left[\left|x^{(i)}(t)-S_{\Delta_{m}}^{(i)}\left(\hat{x}_{m}, t\right)\right|\right] \leqq M_{4+i}(h)^{4-i}, \quad i=0,1,2 .
$$

Proof. Define

$$
\begin{aligned}
e_{i} & =x(j h)-\hat{x}_{i}(j h), \\
e_{i}^{\prime} & =x^{(1)}(j h)-\hat{x}_{i}^{(1)}(j h), \\
E & =\operatorname{col}\left[e_{0}^{\prime}, e_{0}, e_{1}, \cdots, e_{m}, e_{m}^{\prime}\right] .
\end{aligned}
$$

Using these definitions, (5) and (6) give

$$
\begin{aligned}
& e_{m}=\int_{0}^{m h}\left[K(m h, t, x(t))-K\left(m h, t, S_{\Delta_{m}}(x, t)\right)\right] d t \\
& \quad-\int_{0}^{m h}\left[K\left(m h, t, S_{\Delta_{m}}\left(\hat{x}_{m}, t\right)\right)-K\left(m h, t, S_{\Delta_{m}}(x, t)\right)\right] d t, \\
& e_{m}^{\prime}-\left[K(m h, m h, x(m h))-K\left(m h, m h, \hat{x}_{m}(m h)\right)\right] \\
& \quad=\int_{0}^{m h}\left[K^{(1)}(m h, t, x(t))-K^{(1)}\left(m h, t, S_{\Delta_{m}}(x, t)\right)\right] d t \\
& \quad+\int_{0}^{m h}\left[K^{(1)}\left(m h, t, S_{\Delta_{m}}(x, t)\right)-K^{(1)}\left(m h, t, S_{\Delta_{m}}\left(\hat{x}_{m}, t\right)\right)\right] d t
\end{aligned}
$$

From the hypothesis, Lemma 1, and the convergence property of the cubic splines [16, Theorem 2.3.4] we get

$$
\left|\int_{0}^{m h}\left[K^{(i)}(m h, t, x(t))-K^{(i)}\left(m h, t, S_{\Delta_{m}}(x, t)\right)\right] d t\right| \leqq L_{i} M_{7} h^{4}, \quad i=0,1,
$$

and

$$
\begin{aligned}
\left|\int_{0}^{m h}\left[K^{(i)}\left(m h, t, S_{\Delta_{m}}(x, t)\right)-K^{(i)}\left(m h, t, S_{\Delta_{m}}\left(\hat{x}_{m}, t\right)\right)\right] d t\right| & \\
\leqq & \int_{0}^{m h} L_{i}\left|S_{\Delta_{m}}(E, t)\right| d t \\
& \leqq L_{i} M_{1} h\left[\sum_{k=0}^{m-1}\left|e_{k}\right|+\left|e_{m}\right|+\left|e_{m}^{\prime}\right|\right], \quad i=0,1 .
\end{aligned}
$$

Using these, (20) and (21) reduce to the following simultaneous linear difference inequalities:

$$
\begin{aligned}
& \left|e_{m}\right| \leqq L_{0} M_{7} h^{4}+L_{0} M_{1} h\left[\sum_{k=0}^{m}\left|e_{k}\right|+\left|e_{m}^{\prime}\right|\right] \\
& \left|e_{m}^{\prime}\right| \leqq L_{0}\left|e_{m}\right|+L_{1} M_{7} h^{4}+L_{1} M_{1} h\left[\sum_{k=0}^{m}\left|e_{k}\right|+\left|e_{m}^{\prime}\right|\right]
\end{aligned}
$$


Elimination of $e_{m}^{\prime}$ gives

$$
\begin{aligned}
& \left\{\left(1-L_{1} M_{1} h\right)\left(1-L_{0} M_{1} h\right)-L_{0} M_{1} h\left(L_{0}+L_{1} M_{1} h\right)\right\}\left|e_{m}\right| \\
& \quad \leqq\left\{\left(1-L_{1} M_{1} h\right) L_{0} M_{1} h+L_{0} M_{1}^{2} L_{1} h^{2}\right\} \sum_{k=0}^{m-1}\left|e_{k}\right|+\xi_{m},
\end{aligned}
$$

where

$$
\left|\xi_{m}\right| \leqq M_{10} h^{4}
$$

It is easy to observe that, for sufficiently small $h$,

$$
\left\{\left(1-L_{1} M_{1} h\right)\left(1-L_{0} M_{1} h\right)-L_{0} M_{1} h\left(L_{0}+L_{1} M_{1} h\right)\right\} \geqq c>0
$$

and

$$
\left\{\left(1-L_{1} M_{1} h\right) L_{0} M_{1} h+L_{0} M_{1}^{2} L_{1} h^{2}\right\} \leqq M_{11} h .
$$

We note that Eq. (28) implies solvability of $\hat{x}_{m}(m h)$ and consequently of $\hat{x}_{m}^{(1)}(m h)$ from (5) and (6).

Let $\left\{f_{m}\right\}$ be a sequence of real numbers satisfying

$$
f_{m} \leqq \frac{M_{11} h}{c} \sum_{j=1}^{m-1} f_{i}+\xi_{m}
$$

with $f_{0}=0$. Then from (26) and Lemma 2, we get

$$
\left|e_{m}\right| \leqq f_{m} \leqq \xi_{m} \exp \left(M_{11} \cdot s / c\right)=O\left(h^{4}\right)
$$

and hence, from (25),

$$
\left|e_{m}^{\prime}\right| \leqq O\left(h^{4}\right) .
$$

Let $z(t), 0 \leqq t \leqq m h$, be any function such that

$$
z(\cdot) \in C^{5}[0, m h] ; \quad z^{(i)}(k h)=\hat{x}_{k}^{(i)}(k h) ; \quad k=0, \cdots, m ; \quad i=0,1 .
$$

(Note that such a $z(t)$ can be constructed by Hermite interpolation.) Then, by the interpolation property of cubic splines [17],

$$
\begin{aligned}
S_{\Delta_{m}}^{(i)}\left(\hat{x}_{m}, t\right)=z^{(i)}(t)+O\left(h^{4}\right)=x^{(i)}(t)+O\left(h^{4}\right), & \\
& i=0,1, t=h, 2 h, \cdots, m h .
\end{aligned}
$$

Thus, $S_{\Delta_{m}}\left(\hat{x}_{m}, t\right)$ is a cubic spline which, along with its first derivative, agrees with the true solution and its first derivative at the mesh points to the fourth order accuracy. It can then be easily proved (see for example Lemma 4 and Theorem 5 in [12]) that the Theorem holds.

It should be noted that the above Theorem establishes only a convergence result and does not consider the important question of stability. For linear Volterra equations, as stated in the following Corollary, conditions for convergence can be slightly weakened. Also, elimination of $\hat{x}_{m}^{(1)}(m h)$ from Eqs. (5) and (6) is possible, thus simplifying the computation of $S_{\Delta_{m}}\left(\hat{x}_{m}, t\right)$ [i.e. to compute $S_{\Delta_{m}}\left(\hat{x}_{m}, t\right), 0 \leqq t \leqq s$, we can compute $\hat{x}_{1}(h), \cdots, \hat{x}_{m}(m h), \hat{x}_{m}^{(1)}(m h)$ without having to compute all the values $\left.\hat{x}_{1}(h), \hat{x}_{1}^{(1)}(h), \cdots, \hat{x}_{m}(m h), \hat{x}_{m}^{(1)}(m h)\right]$. 
TABLE I

Table for Numerical Examples Investigated

\begin{tabular}{cccc}
\hline Example & $K(t, s)$ & $y(s)$ & Exact Solution $x(s)$ \\
\hline I & $s \cdot t$ & $e^{-s}\left(1+s+s^{2}\right)-s$ & $e^{-s}$ \\
II & $s \cdot t$ & $\sin \pi s\left[1-s / \pi^{2}\right]+\frac{s^{2}}{\pi} \cos \pi s$ & $\sin \pi s$ \\
III & $s^{4} e^{s t}$ & $\left(s-s^{2}\right)+s^{4} e^{s^{2}}\left(1-1 / s^{2}\right)$ & $s$ \\
IV & $s^{4} e^{s t}$ & $\sin \pi s-\frac{s^{4}}{s^{2}+\pi^{2}}$ & $\sin \pi s$ \\
& & $\cdot\left[e^{s^{2}}(s \cdot \sin \pi s)-\pi \cos \pi s\right]+\pi$ & \\
\hline
\end{tabular}

COROLlARY. Let the linear Volterra equation be represented by

$$
x(s)-\int_{0}^{s} \mathrm{~K}(s, t) x(t) d t=y(s), \quad 0 \leqq s \leqq b .
$$

If the solution of (35), $x(\cdot)$, is in $C^{4}[0, m h]$, and

$$
\int_{0}^{m h}\left|\mathbf{K}^{(i)}(m h, t)\right| d t \leqq M_{i}, \quad i=0,1,
$$

then there exist constants $M_{12}, M_{13}, M_{14}$ such that

$$
\sup _{0 \leqq t \leqq m h}\left[\left|x^{(i)}(t)-S_{\Delta_{m}}^{(i)}\left(\hat{x}_{m}, t\right)\right|\right] \leqq M_{12+i}(h)^{4-i}, \quad i=0,1,2,
$$

where $S_{\Delta_{m}}\left(\hat{x}_{m}, t\right)$ is obtained by following the same procedure as before.

Proof. The proof is very similar to that of the Theorem and is therefore omitted.

3. Numerical Examples. In actual computations, two representations of the cubic spline, other than the cardinal spline representation used above, prove to be more helpful. One of them [16] is easily written in terms of $S_{\Delta_{m}}^{(2)}\left(x, t_{i}\right)$ at the mesh points. The other representation developed by Nilson [19] expresses each cardinal spline as a linear combination of two basic cubic arcs. Four examples, shown in Table I, were studied on the interval $[0,1]$. In each case, this interval was subdivided

TABLE II

Maximum Error for the Numerical Examples Investigated

\begin{tabular}{rcccc}
\hline \multicolumn{5}{c}{ EXAMPLE } \\
\multicolumn{1}{r}{$N$} & I & II & III & IV \\
\hline 5 & $3.4351 \times 10^{-5}$ & $1.3814 \times 10^{-4}$ & $6.3846 \times 10^{-4}$ & $7.6124 \times 10^{-4}$ \\
10 & $1.041 \times 10^{-5}$ & $2.4150 \times 10^{-5}$ & $8.7613 \times 10^{-5}$ & $1.3126 \times 10^{-4}$ \\
15 & $7.316 \times 10^{-6}$ & $9.463 \times 10^{-6}$ & $1.127 \times 10^{-5}$ & $4.678 \times 10^{-5}$ \\
20 & $8.743 \times 10^{-8}$ & $1.783 \times 10^{-7}$ & $8.761 \times 10^{-7}$ & $1.389 \times 10^{-6}$ \\
\hline
\end{tabular}


into $N$ equal parts and the solution was obtained recursively starting from $x(0)$ and $x^{(1)}(0)$. Only the maximum error is tabulated for these examples in Table II, but, as noted before, the global approximation could be obtained by the spline interpolation as stated in the text of the paper. Programs for all the four examples were written in double precision using Fortran IV language for a Burroughs B-5500 computer.

4. Conclusions. Global approximations to the solution of Volterra integral equation of the second kind have been obtained. Under appropriate conditions on the kernel and the forcing function, convergence of these approximations to the exact solution and the error bounds have been derived. These results are illustrated by means of numerical examples. Additional smoothness of the approximating solution can be achieved by the use of higher order splines at the cost of increased computation.

5. Acknowledgments. This was a small part of the author's Ph.D. dissertation [20] under Professor Rui J. P. deFigueiredo whose many helpful comments are gratefully acknowledged.

Bell Laboratories

Holmdel, New Jersey 07733

1. A. N. Netravali \& R. J. P. DeFigueiredo, "Spline approximation to the solution of the linear Fredholm integral equation of the second kind". (Submitted for publication.)

2. J. G. JONES, "On the numerical solution of convolutional integral equations and systems of such equations," Math. Comp., v. 15, 1961, pp. 131-142. MR 22 \#12728.

3. B. NOBLE, "The numerical solution of nonlinear integral equations and related topics," Nonlinear Integral Equations (Proc. Advanced Sem. Conducted by Math. Res. Center, U.S. Army, Univ. of Wisconsin, Madison, Wis., 1963), Univ. of Wisconsin Press, Madison, Wis., 1964, pp. 215-318. MR 30 \#3582.

4. M. K. JaIN \& K. D. Sharma, "Numerical solution of linear differential equations and Volterra integral equation using Lobatto quadrature formula," Comput. J., v. 10, 1967, pp. 101-107. MR 35 \#2488.

5. L. Fox \& E. T. Goodwin, "The numerical solution of non-singular linear integral equations," Philos. Trans. Roy. Soc. London Ser. A, v. 245, 1953, pp. 501-534. MR 14, 908.

6. P. LINZ, The Numerical Solution of Volterra Integral Equation by Finite Difference Methods, MRC Technical Report \#827, Madison, Wis., 1967.

7. P. PouzET, Etude, en vue de leur traitement numérique d'équations intégrales et intégro-différentielles du type de Volterra pour des problèmes de conditions initiales, Thesis, University of Strasbourg, 1962 .

8. P. PouzeT, "Méthode d'intégration numérique des équations intégrales et intégrodifférentielles du type de Volterra de seconde espèce. Formules de Runge-Kutta," Symposium on the Numerical Treatment of Ordinary Differential Equations, Integral and IntegroDifferential Equations (Rome, 1960), Birkhäuser, Basel, 1960, pp. 362-368. MR 23 \# B601.

9. M. LAUDET \& H. OulÈs, "Sur l'intégration numérique des équations intégrales du type de Volterra," Symposium on the Numerical Treatment of Ordinary Differential Equations, Integral and Integro-Differential Equations (Rome, 1960), Birkhäuser, Basel, 1960, pp. 117-121. MR 23 \#B597.

10. B. A. BEL'TJUKov, "An analogue of the Runge-Kutta method for the solution of nonlinear integral equations of Volterra type," Differencial'nye Uravnenija, v. 1, 1965, pp. $545-556=$ Differential Equations, v. 1, 1965, pp. 417-433. MR 33, \#3479.

11. A. FELDSTEIN, "Numerical method for nonlinear Volterra integro-differential equations". (Preprint.)

12. F. R. Loscalzo \& T. D. Talbot, "Spline function approximations for solutions of ordinary differential equations," SIAM J. Numer. Anal., v. 4, 1967, pp. 433-445. MR 36 \#4808.

13. F. R. Loscalzo \& I. J. Schoenberg, On the Use of Spline Functions for the Approximation of Solutions of Ordinary Differential Equations, Tech. Summary Report \#723, Math. Res. Center, U.S. Army, University of Wisconsin, Madison, Wis., 1967. 
14. R. S. VARGA, "Error bounds for spline interpolation," Approximations with Special Emphasis on Spline Functions (Proc. Sympos., Univ. of Wisconsin, Madison, Wis., 1969), Academic Press, New York, 1969, pp. 367-388. MR 40 \#6130.

15. H. S. Hung, The Numerical Solution of Differential and Integral Equations by Spline Functions, Tech. Summary Report \#1053, Math. Res. Center, U.S. Army, University of Wisconsin, Madison, Wis., March 1970.

16. J. H. Ahlberg, E. N. Nilson \& J. L. Walsh, The Theory of Splines and Their Applications, Academic Press, New York, 1967. MR 39 \#684.

17. G. BIRKHOFF \& C. R. DE BoOR, "Piecewise polynomial interpolation and approximation," Approximation of Functions (Proc. Sympos. General Motors Res. Lab., 1964), Elsevier, Amsterdam, 1965, pp. 164-190. MR 32 \#6646.

18. M. LEES, "Approximate solutions of parabolic equations," J. Soc. Indust. Appl. Math., v. 7, 1959, pp. 167-183. MR 22 \#1092.

19. E. N. Nilson, "Cubic splines on uniform meshes," Comm. ACM, v. 13, 1970, pp. 255-258.

20. A. N. Netravali, Signal Processing Techniques Based on Spline Functions, Ph.D. Dissertation, Rice University, Houston, Texas, 1970. 